\title{
Analysis of the fractures of metallic materials using optical coherence tomography
}

\author{
Gh. Hutiu ${ }^{1}$, V.-F. Duma ${ }^{* 1,2}$, D. Demian ${ }^{1}$, A. Bradu ${ }^{3}$, A. Gh. Podoleanu ${ }^{3}$ \\ 13OM Optomechatronics Group, Aurel Vlaicu University of Arad, 77 Revolutiei Ave., 310130 Arad, \\ Romania \\ ${ }^{2}$ Doctoral School, Polytechnic University of Timisoara, 1 Mihai Viteazu Ave., 300222 Timisoara, \\ Romania \\ ${ }^{3}$ Applied Optics Group, School of Physical Sciences, University of Kent, Canterbury, CT2 7NH, UK
}

\begin{abstract}
Forensic in situ investigations, for example for aviation, maritime, road, or rail accidents would benefit from a method that may allow to distinguish ductile from brittle fractures of metals - as material defects are one of the potential causes of such accidents. Currently, the gold standard in material studies is represented by scanning electron microscopy (SEM). However, SEM are large, lab-based systems, therefore in situ measurements are excluded. In addition, they are expensive and time-consuming. We have approached this problem and propose the use of Optical Coherence Tomography (OCT) in such investigations in order to overcome these disadvantages of SEM. In this respect, we demonstrate the capability to perform such fracture analysis by obtaining the topography of metallic surfaces using OCT. Different materials have been analyzed; in this presentation a sample of low soft carbon steel with the chemical composition of C $0.2 \%$, Mn $1.15 \%$, S $0.04 \%$, P $0.05 \%$ and Fe for the rest has been considered. An in-house developed Swept Source (SS) OCT system has been used, and height profiles have been generated for the sample surface. This profile allowed for concluding that the carbon steel sample was subjected to a ductile fracture. A validation of the OCT images obtained with a 10 microns resolution has been made with SEM images obtained with a $4 \mathrm{~nm}$ resolution. Although the OCT resolution is much lower than the one of SEM, we thus demonstrate that it is sufficient in order to obtain clear images of the grains of the metallic materials and thus to distinguish between ductile and brittle fractures. This study analysis opens avenues for a range of applications, including: (i) to determine the causes that have generated pipe ruptures, or structural failures of metallic bridges and buildings, as well as damages of machinery parts; (ii) to optimize the design of various machinery; (iii) to obtain data regarding the structure of metallic alloys); (iv) to improve the manufacturing technologies of metallic parts.
\end{abstract}

Keywords: Optical Coherence Tomography (OCT), Scanning Electron Microscopy (SEM), metallic materials, surface topography, interferometric imaging, forensic investigations.

\section{INTRODUCTION}

Various methods and types of equipment are currently used in order to analyze the structure of metallic materials. Thus, structural images are obtained using magnifying lens, ordinary optical microscopes, as well as optical microscopes functioning at high temperatures, transmission electron microscopes (TEM), scanning electron microscopes (SEM), reflection electron microscopes, field ion microscopes, or atomic force microscopes (AFM) [1, 2].

The diffraction pattern of the crystal lattice is analyzed by means of X-ray equipment, low electron diffraction equipment, neutron diffraction equipment, etc. Material composition is analyzed by means of electron microprobes (with electron beams and X-rays), ion microprobes, photoelectron spectrometers, Auger electron spectrometers, etc.

\footnotetext{
*Email: duma.virgil@osamember.org; Phone: +40-751-511451; site: http://3om-group-optomechatronics.ro/
} 
Laboratories currently use all these kinds of equipment capable to determining images of structure, the diffraction patterns and the composition of different materials.

Another method capable of performing material studies, in a non-invasive manner, with micrometer resolutions and with millimeter-depth penetration is Optical Coherence Tomography (OCT) [3-5]. Beside its use in biomedical imaging, OCT has been used to imagine a range of materials, including:

(i) Plastics and composites [6-10]: their analysis focused on internal structure, matrices and reinforcement, superficial stress of resin drops, layer thickness, as well as defects/ flaws occurring inside layers.

(ii) Electronic materials [11] analyses were carried out concerning thickness of layer, layer flaws/defects, and defects occurring between layers.

(iii) Paintings [12], in order to analyze structure of paint layers, thickness of layers, as well as sketches existing below paint layers

(iv) Dental materials [13-16] have been investigated for the layer/stratum thickness, inside layer defects, and interlayer defects and layer contraction.

We have also approached metallic materials studies using OCT [17], in order to analyze their structure, and more specific their fracture surfaces, after being previously subjected to tests for ductile or brittle fractures. This is an essential issue, as the major aims of fracture surface analysis are to determine the causes that generate damage of machinery parts, to obtain new information regarding the structure of solid bodies, to optimize machinery design, and to improve metallic material manufacturing technologies.

In general, metallic material fractures can be classified according to their deformation at failure, into ductile, brittle, and fatigue fractures. From the point of view of the crystallographic manner in which the fracture occurs, i.e., sliding or cleavage, the appearance of the fracture can be of the fiber or fiery type.

Brittle fractures of metallic materials are achieved by cleavage and consists in the breakdown of atomic bonds between atoms situated on two adjacency planes perpendicular to the direction in which the normal tensile stress applies. Cleavage fracture may occur through the crystal grain (fiery aspect) or at crystal grains boundaries (fiber aspect). The surface of granular fractures is plane and perpendicular to the direction in which tensile stress applies. Due to the fact that in polycrystalline materials cleavage planes in each grain are not always perpendicular to the direction of force (as grain axes are differently oriented), at microscopic scale fracture surfaces are not perfectly plane, except for the grain surface.

Ductile fractures generate less serious problems than brittle fracture under operating conditions. Ductile fracture (or shearing fracture) is produced inside the crystal grains in sliding planes with maximum atom density. The fracture crack propagates along the maximum tangential stress of the load applied. The crack moves under a $45^{\circ}$ angle from the tensile stress applied.

Fatigue fractures occur when metallic materials are subjected to variable loads at high amplitudes. As a result, the resistance and the ductility of metallic materials decrease, and fracture occurs at lower tensile stress values than their tensile strength or even than their flow stress. When fatigue fracture occurs in metallic materials, three areas can be distinguished at microstructure level: the first area is the crack initiation one - for which the crack propagates in ductile manner, expanding over several grains;-the second area is the fatigue fracture one, which displays fatigue lines called streaks, where the fracture crack passes through the grains and displays a fiery aspect at macroscopic level; the third area is that of the final fracture which can be only brittle. Fatigue fracture is thus brittle-like even in normally ductile materials. It is worth highlighting that more than $90 \%$ of the failures occurring under operating/working conditions are due to fatigue.

The present paper reports on the analyses of surface topography of metallic materials, as studied in detail in [17]. A steel sample subjected to ductile fracture is considered as an example in the present paper. The tests carried out have been demonstrated that the OCT method is capable to replace the gold standard in this field, i.e., the SEM method, that has much higher resolutions, but also higher cost, and it is labor and time consuming; it is also strictly lab-based, in comparison with OCT, which can work as mobile units equipped with handheld scanning probes with galvoscanners [15, 16] or Micro-Electro-Mechanical Systems (MEMS) [18], in order to perform in situ, industrial or forensic investigations. 


\section{METHODS AND MATERIALS}

\subsection{Methods}

The surface microstructures were analyzed using two different pieces of equipment, a $10 \mu \mathrm{m}$ resolution swept source (SS)-OCT system and a $4 \mathrm{~nm}$ resolution SEM.

A schematic diagram of the SS-OCT imaging system has been provided in Fig. 1, [17]. It includes as the optical source, an SS (Axsun Technologies, Billerica, Massachusetts), with a central wavelength of $1060 \mathrm{~nm}$, a sweeping range of 106 $\mathrm{nm}$ (quoted at $10 \mathrm{~dB}$ ), and a $100 \mathrm{kHz}$ line rate. Its anatomy has been described in detail in [17]. For the SEM analysis a high vacuum FEI Quanta 250 SEM and a secondary Everhard-Thomley electron detector were used. The samples have not been metal coated. The working parameters (i.e., WD, working distance, and Pa, pressure) may vary depending on the image selected.

The samples were inserted in the microscope and examined at various magnitudes. They were mounted on a copper or aluminum conductive holder stub using carbon wafers with adhesive on both sides. The mounting of the samples was done using the lens of a binocular microscope in order to expose the investigated area directly to the electron beam scanning the sample. The sample alignment on the stub is important in this stage in order to reduce the tilting inside the microscope.

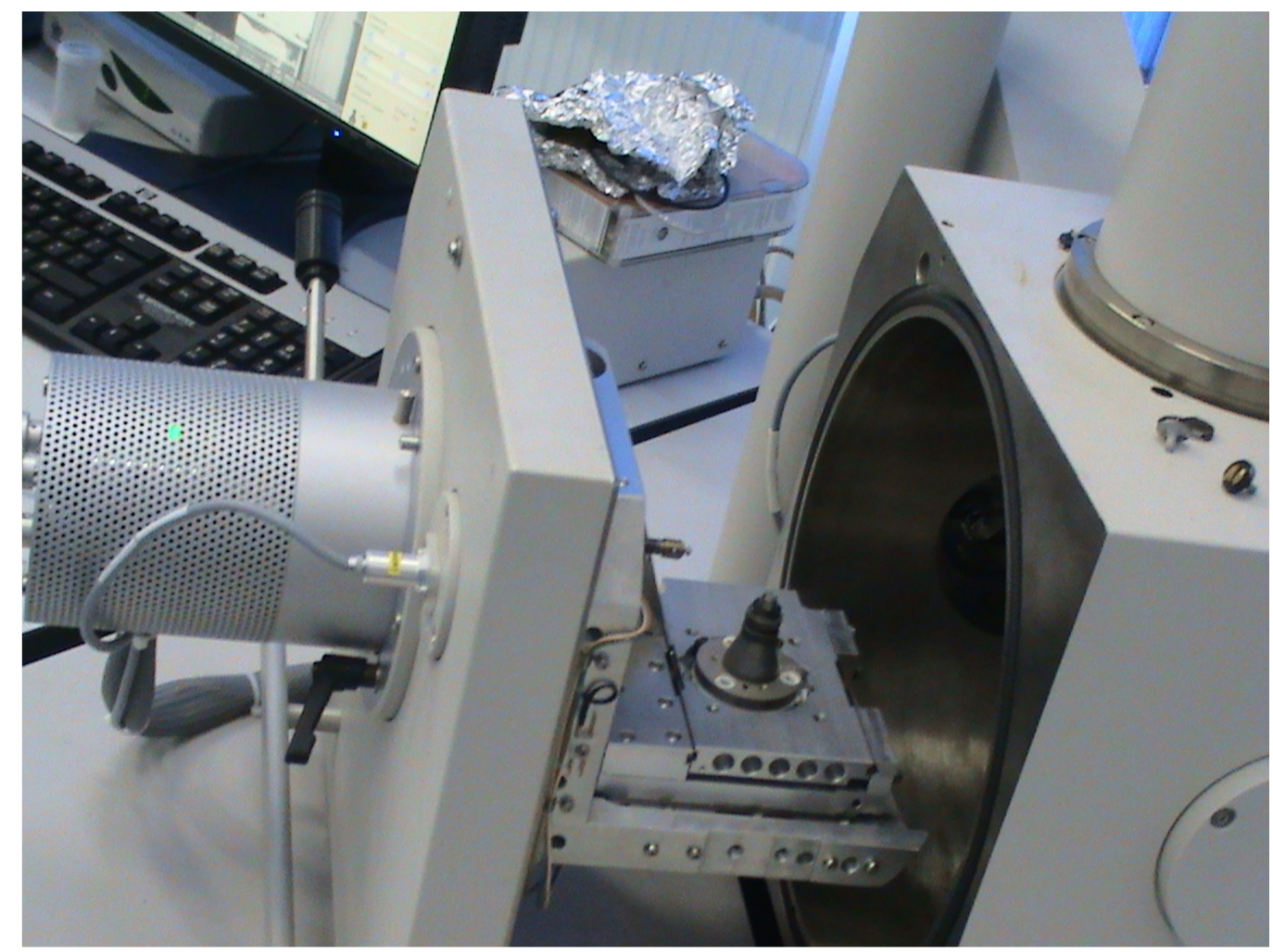

Figure 1. Scanning Electron Microscope (SEM) utilized in the investigations, including in those presented in [17], with the metallic sample positioned in its holder.

\subsection{Materials analyzed}

In order to demonstrate the utility of the OCT method for fracture analysis, we have considered in [17] both ductile and brittle fractures $[19,20]$. In the following a different steel sample (OL44) than the ones previously investigated is presented; such a material is usually subjected to ductile fractures. 
The microstructure of this steel has been analyzed after its subjection to fracture tests. OL 44 (STAS 500/2-80) has the following chemical composition: $\mathrm{C} 0.2 \%, \mathrm{Mn} 1.15 \%, \mathrm{~S} 0.04 \%, \mathrm{P} 0.05 \%$, and Fe for the rest. This steel is used for welded structures (cranes, car frames, etc). Its microstructure is made of ferrite and pearlite grains.

\subsection{Sample processing method}

The OL 44 steel sample was subjected to tensile tests. Due to its chemical composition and microstructure this type of steel always breaks with a ductile fracture.

After the testing of the sample, a $5 \mathrm{~mm}$ high part containing the cup fracture was examined using both OCT and SEM, the latter mainly for the validation of the OCT images. The lateral margin of the sample was marked so as to capture the same zone with both methods. Despite the difference in resolution between the two methods, the images generated by the OCT are shown to be compatible with the images generated by the SEM.

\section{RESULTS}

Fig. 2 shows the fracture surface of low carbon steel sample OL 44.

Fig. 2(a) shows the overall image of the OL 44, and the ductile fractured sample. It can be noticed that the sample has a cup-type shape fracture that is characteristic for a ductile fracture.

Fig. 2(b) shows the fracture image for steel 44 generated by SEM. It can be noticed that all the grains broke in a transgranular manner, which is a characteristic of the ductile sharing fracture. As the grains have different orientations against the applied load, it can be noticed that only few grains were broken under a $45^{\circ}$ angle from the tensile stress applied.

Fig. 2(c) shows the image of the broken surfaces of OL 44 generated via OCT. The same area as the one selected in Fig. 2(b) for SEM has been imaged in Fig. 2(c) with OCT. In order to demonstrate that the OCT images are similar to those generated via SEM, some grains have also been numbered on both images, SEM and OCT. Both images clearly show that the grains broke in a transgranular manner.

\section{CONCLUSIONS}

1) The images acquired in [17] and in this study show the fracture surfaces for different metallic materials. In this study a steel sample which broke in a ductile manner has been shown. These images were generated using two types of technologies, OCT and SEM.

2) Analyzing the images generated via SEM at a $4 \mathrm{~nm}$ resolution and that of the images generated via OCT at a $10 \mu \mathrm{m}$ overall resolution it can be concluded that the OCT images are compatible with the SEM images for this type of application. The present study demonstrates that OCT can replace the SEM in the analysis of metallic surfaces broken in both a ductile and a brittle manner.

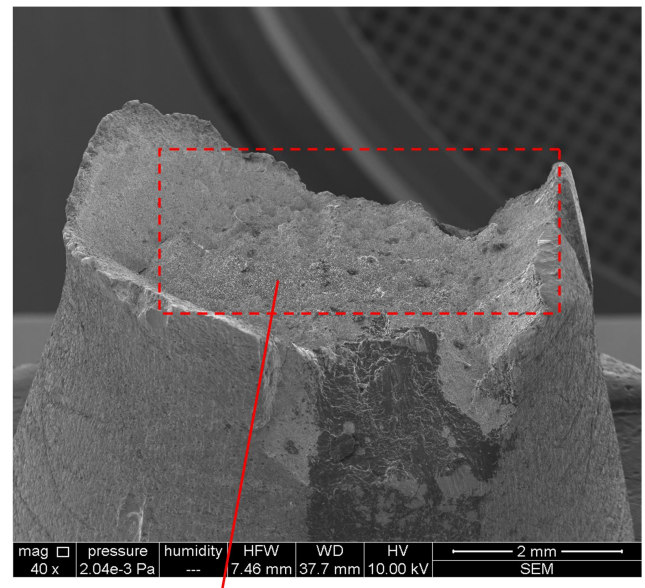

(a)

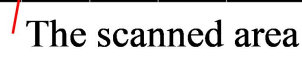




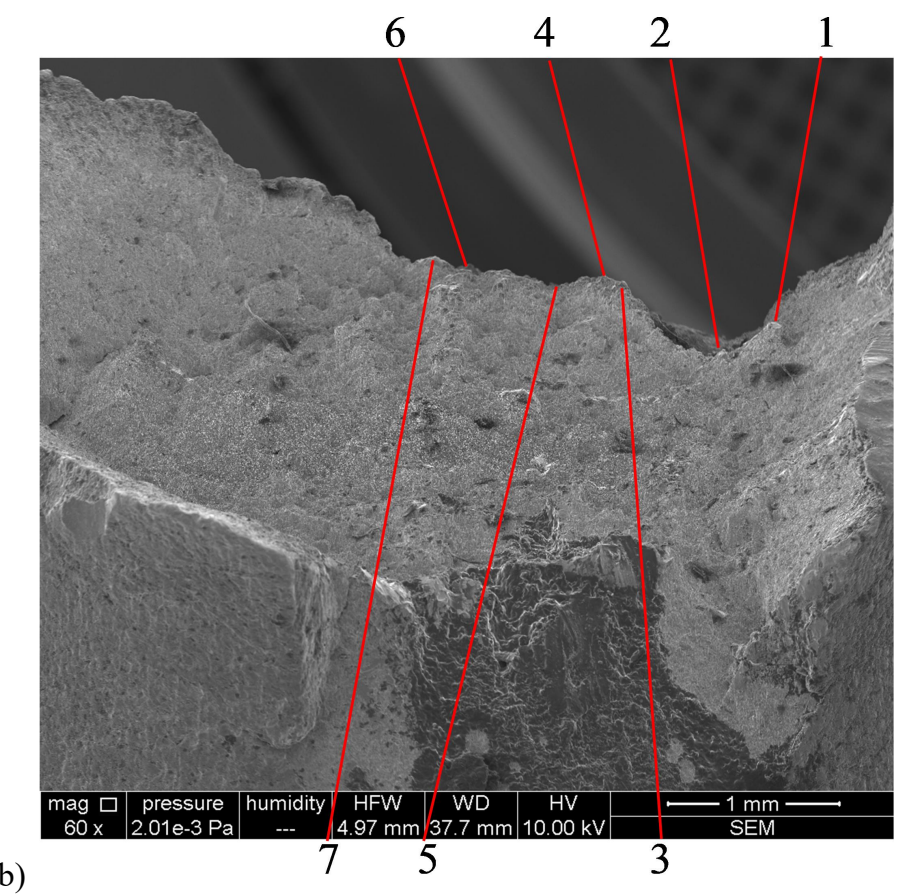

(b)

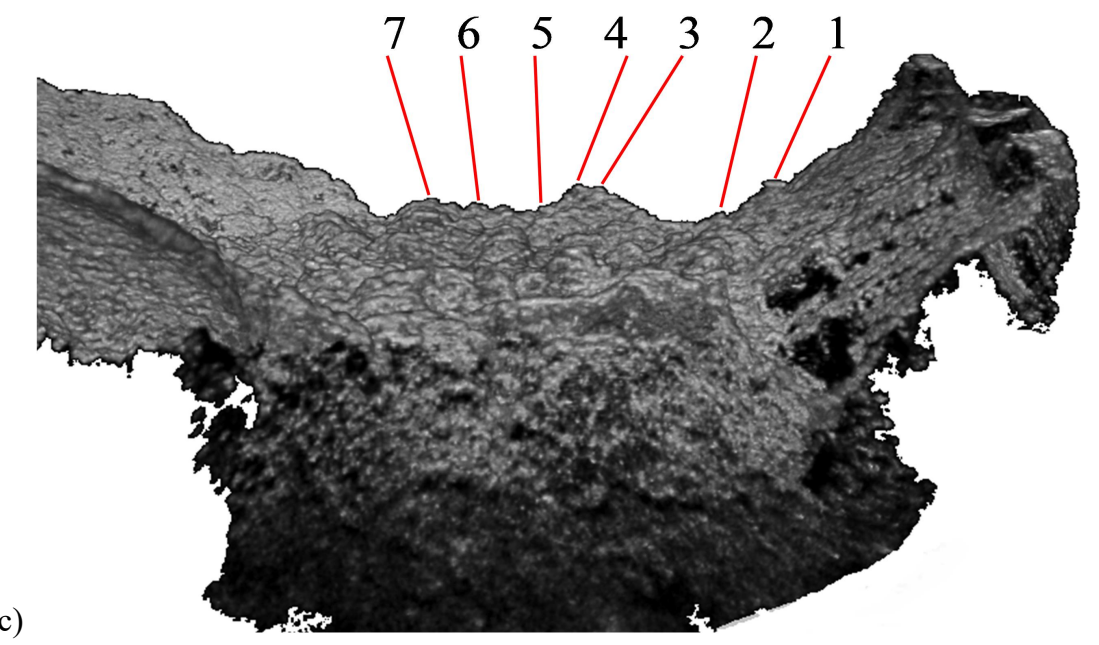

Figure 2 Imaging the fracture of OL 44 steel: (a) frontal SEM overview of the entire sample; (b) SEM image; (c) OCT image of the same area.

3) Another advantage of the OCT method as compared to the SEM is that the former can generate the image of the entire sample surface through consecutive scanning operations using an OCT mobile unit and handheld scanning probes [15, 16]. These scanning operations do not destroy the metallic parts, which is an essential issue in the case of aviation, maritime, road or rail accidents, etc. In such cases, the broken parts must stay intact.

\section{ACKNOWLEGMENTS}

This work was funded by the Romanian National Authority for Scientific Research, through a Transfer to Industry CNDI-UEFISCDI project PN-III-P2-2.1-PTE-2016-0181 (http://3om-group-optomechatronics.ro/). 


\section{REFERENCES}

[1] Goldstein, J., Newbury, D. E., Lyman, C. L., Echlin, P., Lifshin, E., Sawyer, L., [Scanning Electron Microscopy and X-ray Microanalysis], Third Edition, Springer Science + Business Media Inc., 128-192 (2003).

[2] Echlin, P., [Handbook of Sample Preparation for Scanning Electron Microscopy and X-Ray Microanalysis], Springer Science + Business Media Inc., 19-65 (2009).

[3] Huang, D., Swanson, E. A., Lin, C. P., Schuman, J. S., Stinson, W. G., Chang, W., Hee, M. R., Flotte, T., Gregory, K., Puliafito, C. A., and Fujimoto, J. G., "Optical coherence tomography," Science 254(5035), 1178-1181 (1991).

[4] Wojtkowski, M., "High-speed optical coherence tomography: basics and applications," Applied Optics 49, D30-D61 (2010).

[5] Podoleanu, A. Gh. and Rosen, R. B., "Combinations of techniques in imaging the retina with high resolution," Progress in Retinal and Eye Research 27, 464-499 (2008).

[6] Awaja, F., Arhatari, B., Wiesauer, K., Leiss, E., Stifter, D., "An investigation of the accelerated thermal degradation of different epoxy resin composites using X-ray microcomputed tomography and optical coherence tomography," Polymer Degradation and Stability 94, 1814-1824 (2009).

[7] Manukyan, S., Sauer, H. S., Roisman, I. R.., Baldwin, K. A., Fairhurst, D.F., Liang, H., Venzmer, J., Tropea, C., "Imaging internal flows in a drying sessile polymer dispersion drop using Spectral Radar Optical Coherence Tomography (SR-OC)," Journal of Colloid and Interface Science (2013).

[8] Price, J. H. P., Feng, X., Heidt, A. M., Brambilla, Horak, G. P, Poletti, F., Ponzo, G., Petropoulos, P., Petrovich, M., Shi, J., Ibsen, M., Loh,W. H., Harvey N. Rutt, H. N., Richardson, D. J., "Supercontinuum generation in non-silica fibers," Optical Fiber Technology 18, 327-344 (2012).

[9] Jonathan, E., "Non-contact and non-destructive testing of silicon V-grooves: A non-medical application of optical coherence tomography (OCT)," Optics and Lasers in Engineering 44, 1117-1131 (2006).

[10] Nemeth, R., Gahleitner, A., Hannesschlager, G., Giselher Pfandler, G., Leitner, M., "Ambiguity-free spectraldomain optical coherence tomography for determining the layer thicknesses in fluttering foils in real time," Optics and Lasers in Engineering 50 (2012).

[11] Serrels, K. L., Renner, M. K., Reid, D. T., "Optical coherence tomography for non-destructive investigation of silicon integrated-circuits," Microelectronic Engineering 87, 1785-1791 (2010).

[12] Liang, H., Gomez Cid, M., Cucu, R. G., Dobre, G. M., Podoleanu, A. G., Saunders, D., "En-face optical coherence tomography - a novel application of non-invasive imaging to art conservation, "Optical Society of America, (2005).

[13] Oancea, R., Bradu, A., Sinescu, C., Negru, R. M., Negrutiu, M. L., Antoniac, I., Duma, V.-F., and Podoleanu, A. Gh., "Assessment of the sealant/tooth interface using optical coherence tomography," J. of Adhesion Science and Technology 29(1), 49-58 (2015).

[14] Sinescu, C., Negrutiu, M. L., Bradu. A., Duma, V.-F., and Podoleanu, A. Gh., "Noninvasive quantitative evaluation of the dentin layer during dental procedures using Optical Coherence Tomography," Computational and Mathematical Methods in Medicine, Paper ID 709076 (2015).

[15] Demian, D., Duma, V.-F., Sinescu, C., Negrutiu, M. L., Cernat, R., Topala, F. I., Hutiu, Gh., Bradu, A., and Podoleanu, A. Gh., "Design and testing of prototype handheld scanning probes for optical coherence tomography," Journal of Engineering in Medicine 228(8), 743-753 (2014).

[16] Duma, V.-F., Dobre, G., Demian, D., Cernat, R., Sinescu, C., Topala, F. I., Negrutiu, M. L., Hutiu Gh., Bradu, A., and Podoleanu, A. Gh., "Handheld scanning probes for optical coherence tomography," Romanian Reports in Physics 67(4), 1346-1358 (2015).

[17] Hutiu Gh.,_Duma V.-F., Demian D.,_Bradu A., and Podoleanu A. Gh., "Surface imaging of metallic material fractures using optical coherence tomography,"_Applied Optics_53(26), 5912-5916 (2014).

[18] Cogliati, A., Canavesi, C., Hayes, A., Tankam, P., Duma, V.-F., Santhanam, A., Thompson, K. P., and Rolland, J. P., "MEMS-based handheld scanning probe with pre-shaped input signals for distortion-free images in Gabor-Domain Optical Coherence Microscopy," Opt. Express 24(12), 13365-13374 (2016).

[19] Ruggieri, C., Dodds, R. H., "A transferability model for brittle fracture including constraint and ductile tearing effects: probabilistic approach," International Journal of Fracture 34(4), 309-340 (1996).

[20] Honomura, T., Yin, F., Nagai, K., "Ductile-brittle transition temperature of ultrafine ferrite/cementite microstructure in low carbon steel controlled by effective grain size," ISIJ 44 (3), 610-617 (2004). 